\title{
The effect of therapy on TRM in psoriatic lesions
}

\author{
Agnieszka Owczarczyk-Saczonek ${ }^{1}$, Marta Kasprowicz-Furmańczyk ${ }^{1}$, Joanna Czerwińska ${ }^{1}$, \\ Magdalena Krajewska-Włodarczyk², Waldemar Placek ${ }^{1}$
}

${ }^{1}$ Department of Dermatology, Sexually Transmitted Diseases and Clinical Immunology, University of Warmia and Mazury, Olsztyn, Poland ${ }^{2}$ Rheumatology Clinic, University of Warmia and Mazury, Olsztyn, Poland

Adv Dermatol Allergol 2022; XXXIX (1): 209-220

DOI: https://doi.org/10.5114/ada.2021.113125

\begin{abstract}
Introduction: The course of psoriasis is associated with recurrence of the lesions at the same location despite effective treatment. It is due to the presence of TRM (tissue-resident memory cells) in the seemingly healthy skin, which may initiate an inflammatory cascade.

Aim: The assessment of TRM in psoriatic lesions prior to and after 12 weeks of systemic therapy with methotrexate (MTX) or secukinumab (SEC) or ixekizumab (IXE) or adalimumab (ADA).

Material and methods: TRM markers (CD4, CD8, CD103, CD69, CD49, CXCR6) and the tissue expression of cytokines (IL-17, IL-22) in the psoriatic lesions obtained from 13 patients compared to 10 healthy skin samples were evaluated with immunohistochemistry. Biopsy specimens were collected three times from the same psoriatic plaque before and after 4 and 12 weeks of therapy.

Results: The expression of TRM markers in the lesions decreased at three time points (W0, W4, W12), revealing the diminished intensity of fluorescence over time with each therapy. The most rapid response was observed with anti-IL-17 therapy at W4 of treatment, while with MTX and ADA at W12.

Conclusions: The decreased expression of TRM markers occurring predominantly in the lesional dermis and not in the epidermis over 12 weeks of observation may be due to the poorer penetration of systemic drugs to the epidermis, or the process of psoriatic lesion regression in the epidermis is secondary to the reduction of inflammation in the skin, or TRM in the epidermis may be more resistant to therapy.
\end{abstract}

Key words: psoriasis, tissue-resident memory cells, methotrexate, biologics.

\section{Introduction}

The course of psoriasis is associated with the persistence of lesions at specific locations and their recurrence at the same location (up to $90 \%$ of eruptions) despite effective treatment. According to the literature, after the regression of psoriatic plaques, a trace of inflammation remains in the seemingly healthy skin in the form of tissueresident memory (TRM) cells. The cells constitute a trace of "immunological memory". They may rapidly induce inflammation triggering the recurrence of the disease [1, 2].

TRM cells are unable to recirculate. In psoriasis they are represented by two main types: TRM CD8+CD69+CD103+ abundant in the lesional epidermis, and TRM CD4+ CD69+CD103+ located in the dermis [1, 3]. Epidermal TRM CD8+ express CD103, a receptor of adhesion for E-cadherin, which determines their location in the epithelial tissue [3-7]. The CD69 antigen plays a key role in differentiating $T$ cells in tissues from those in circulation. It is responsible for their settling, preventing their recirculation via the suppression of the sphingosine 1-phosphate receptor (S1P1). It enables the differentiation between TRM CD69+ and TEM (effector memory) cells which are negative (CD69-) [8]. TRM cells also express CD49a molecule which binds collagen IV present in the stratum basale [6, 9-11]. CD103+ TRM cells produce IFN- $\gamma$, IL-17A and IL-22 [3]. As regards ex vivo-multiplied T lymphocytes some populations of CD8+CD103+ T lymphocytes produce IFN- $\gamma$, IL-17A or IL-22, while CD4+CD103+ $\mathrm{T}$ lymphocytes rarely produce the cytokines. As regards CD8+ T lymphocytes, IL-17A is more commonly produced by TRM CD103+ cells than by CD103- T lymphocytes. Therefore, CD8+CD103+ TRM cells are effective in producing IL-17A. Other molecules, discerning TRM cells from other circulating types of T memory cells, includ-

Address for correspondence: Agnieszka Owczarczyk-Saczonek MD, PhD, Department of Dermatology, Sexually Transmitted Diseases and Clinical Immunology, University of Warmia and Mazury, Al. Wojska Polskiego 30, 10-229 Olsztyn, Poland, phone: +48 896786670 , fax: +48 8967866 41, e-mail: aganek@wp.pl

Received: 1.09.2021, accepted: 21.11.2021. 
ing CXCR3, CXCR6 and CD101 were also described in the literature [10-12]. It was determined that CD49a, CD103, CXCR6, CX3CR1 and PD-1 were the essential TRM surface markers which differentiated CD69+ and CD69- human memory cells in tissues [8, 11]. However, some researchers consider CXCR6 as a TRM-specific marker present in the mucous membrane and lymphatic tissue [11].

The particular pathogenic properties of TRM CD8+ in psoriasis are due to the fact that they express the IL-23 receptor and are capable of producing proinflammatory IL-17 and IL-22 in the skin, even many months after the resolution of lesions, and their quantity is correlated with the duration of the disease [1, 2, 4, 6, 9, 13-15]. It also explains the rapid recurrence of lesions at specific locations after a trigger factor is activated.

The implementation of treatment, both systemic and topical, aims at the reduction of inflammation, suppression of the production of TRM and the limitation of the number of TRM cells. It may be of key importance in the explanation of rapid recurrences after topical glucocorticoid (GCS) use and longer remission rates after the administration of systemic preparations [16, 17]. The duration of remission varied even in case of treatment with monoclonal antibodies. The use of IL-23 inhibitors proved to result in longer psoriasis remission after treatment discontinuation compared to TNF inhibitors [3, 18]. TRM quantity is not only influenced by the type of the drug and its mechanism of action, but also by the duration of therapy necessary for the quantity to be reduced. The issue has not been fully elucidated and requires further research.

\section{Aim}

The present study aimed at the assessment of the occurrence of TRM in psoriatic eruptions before and after therapy in patients treated systemically with methotrexate (MTX) or secukinumab (SEC) or ixekizumab (IXE) or adalimumab (ADA).

\section{Material and methods}

The study group included patients with plaque psoriasis $(n=13)$, aged 18 to 60 with no concomitant psoriatic arthritis. The patients were treated in our centre. The exclusion criteria were as follows: chronic and acute inflammatory conditions other than psoriasis, neoplastic disease, exacerbation of a cardiovascular disease, cardiac, renal or liver failure. The inclusion criteria were as follows: patients with the severity of psoriatic lesions PASI > 10, no systemic treatment of psoriasis for at least 4 weeks or topical treatment for 1 week prior to enrolment in the study.

The control group $(n=10)$ included healthy volunteers with no previous/family history of psoriasis, no concomitant autoimmune or inflammatory diseases.

Psoriatic patients were treated with MTX at a dose of $15 \mathrm{mg} /$ week for 12 weeks. They also received folic acid
$5 \mathrm{mg}$ on the second day after MTX administration $(n=4)$. Anti-IL-17 drugs $(n=5)$ : secukinumab and ixekizumab, and anti-TNF drugs $(n=4)$ were administered according to recommended therapeutic regimens.

Biopsy specimens were collected three times from the same psoriatic plaque before introducing the therapy, after 4 and 12 weeks of therapy initiation (with the use of a 4-mm biopsy punch).

Tissue samples (lesional skin obtained from psoriatic patients and non-lesional skin from healthy individuals) were cut into $5-\mu \mathrm{m}$-thick sections with the use of the cryostat system (SLEE MEV, Germany) and mounted onto glass slides coated with poly-L-lysine (Menzel-Glaser, Braunschweig, Germany). Frozen sections of the examined tissues were thawed to room temperature and fixed in acetone. After being rinsed in $0.01 \mathrm{M} \mathrm{PBS}$, they were incubated with $2.5 \%$ normal horse serum for $30 \mathrm{~min}$ at room temperature (Vector Laboratories, USA) to decrease nonspecific binding. Subsequently, they were incubated at $4^{\circ} \mathrm{C}$ overnight with mouse anti-CD8 and CD4 or rabbit anti-CD103, CD69, CD49, CXCR6, IL-17 and IL-22 polyclonal antibodies (1: 50; Biorbyt, UK). On the following day, the sections were washed 3 times in PBS and incubated for 30 min with secondary horse anti-mouse/ rabbit antibodies (commercially diluted; ImmPRESS Universal reagent Anti-Mouse/Rabbit Ig; Vector Laboratories). During the analysis of CD4+CD103+ and CD8+CD103+, after being blocked with $2.5 \%$ normal goat serum for $30 \mathrm{~min}$, the frozen sections were incubated with the primary mouse anti-CD8 and-CD4 and rabbit anti-CD103 polyclonal antibody (1:50; Biorbyt, UK) and the secondary goat anti-mouse/rabbit antibodies (VectaFluor Duet Kit, X-Rabbit IgG/D488 X-Mouse IgG, Vector Laboratories, USA). The immunohistochemical specimens were viewed under a fluorescent microscope ( $\mathrm{CH} 30 / \mathrm{CH} 40$; Olympus, Tokyo, Japan).

The study was conducted according to the guidelines of the Declaration of Helsinki, and approved by the Bioethical Committee of the Warmia and Mazury University (24/2020).

\section{Statistical analysis}

After the assumptions of the normality and homogeneity of variances were checked, the results were processed statistically with non-parametric tests (the system was not in accordance with normal distribution). ANOVA Kruskal-Wallis test was used to compare the studied groups with the controls and Friedman ANOVA (Kendall's W Value) was used to compare repeatable measurements at three time points during different types of therapy in the studied groups. Correlation between proteins was analysed with the Spearman's test. All statistical analyses were performed with Statistica software (release 13, StatSoft Inc., USA). Differences were regarded as statistically significant at $p<0.05$. The results were expressed as means \pm SEM. 


\section{Results}

The immunoreactive area of CD8 was always larger in the epidermis than in the dermis $(p<0.05)$ of patients undergoing therapy with anti-interleukin 17 (SEC or IXE) (Figure 1). During the $4^{\text {th }}$ week of observation, the decrease in CD8 immunoreactive area was observed in both skin layers $(p<0.05)$ with the levels being comparable to those in healthy individuals. Similarly, the decrease in CD4 immunoreactive area was noted both in the epidermis and in the dermis during the second measurement $(p<0.05)$, but the immunoreactive areas were larger in the dermis during the therapy $(p<0.05)$. The areas of CD103 and CD49 remained unchanged during the therapy, but a higher level was observed in the epidermis at each time point $(p<0.05)$. In case of CD69, the area diminished in the epidermis during the $4^{\text {th }}$ week of the therapy and then it remained unchanged until the $12^{\text {th }}$ week $(p<0.05)$. No changes were observed in the dermis between measurements. Contrary to CD69, the area of CXCR6 did not change in the epidermis, whereas in the dermis it was smaller during the $4^{\text {th }}$ and $12^{\text {th }}$ week than before the therapy $(p<0.05)$. The immunoreactive area of IL-17A and IL-22 was stable in the epidermis during the therapy. However, a significant decrease was noted in the dermis during the $4^{\text {th }}$ week $(p<0.05)$, which was equal to the outcomes of healthy controls. The level of IL-22A in the epidermis correlated with CD103 $(r=0.6257$, $p<0.05)$ and CD69 $(r=0.6250, p<0.05)$ during the therapy with anti-IL-17.

No changes in the immunoreactive areas of CD8, CD103 and CD49 were reported in the second analysed therapy with anti-tumour necrosis factor $\alpha$ (ADA) (Figure 2). The areas were larger in the epidermis in comparison with the dermis $(p<0.05)$. Interestingly, in case of CD4, the decrease in area size was observed during the third measurement (the $12^{\text {th }}$ week) both in the epidermis as well as in the dermis $(p<0.05)$. The immunoreactive area of CD69 and CXCR6 $(p<0.05)$ in the epidermis was decreased during the $12^{\text {th }}$ week of observation. As regards the dermis, the area diminished only in case of CD69. In contrast, the area of IL-17A and IL-22 remained unchanged in the epidermis, but it was smaller in the dermis during the $4^{\text {th }}$ week of observation than before the therapy $(p<0.05)$.

Similarly to the therapy with anti-IL-17, the immunoreactive area of CD8 was decreased in the epidermis during therapy with MTX. However, the response was delayed (the $12^{\text {th }}$ week of therapy, $p<0.05$ ) (Figure 3 ). The change during the $12^{\text {th }}$ week was also noted in case of CD4 area, but it was observable in the dermis $(p<0.05)$. As regards the third measurement, the decrease in CD103 and CD69 was reported in the epidermis $(p<0.05)$, whereas the area in the dermis was unchanged. The area of CD49 and CXCR6 was constant during therapy, but differences between layers were observed $(p<0.05)$. In con- trast, the area of IL-17A remained unchanged in the epidermis. However, a significant decrease was observed in the dermis during the $4^{\text {th }}$ week of the therapy $(p<0.05)$. Similarly, in case of IL-22, the area in the dermis was reduced $(p<0.05)$ during the $4^{\text {th }}$ week (equating to the outcome in healthy controls). Additionally, the decrease in IL-22 was also noted in the epidermis during the $12^{\text {th }}$ week $(p<0.05)$. The changes of the area of CD8 were negatively correlated with IL-17A $(r=-0.8461, p<0.05)$ in the epidermis.

The location of typical TRM markers in the lesional epidermis and dermis was assessed at three time points for all the therapies. It was demonstrated that the intensity of marker fluorescence diminished throughout the therapies (Figure 4, Table 1).

The analysis of CD4+CD103+ and CD8+CD103+ during different systemic therapies showed changes in areas only in case of treatment with anti-IL-17. The decrease in the size of areas was reported only in the epidermis during the $4^{\text {th }}$ week $-C D 4+C D 103+(p<0.05)$ and during the $12^{\text {th }}$ week $-C D 8+C D 103+(p<0.05)$, in case of the absence of changes in the dermis (Figures 5 and 6).

\section{Discussion}

Similarly to other authors, we demonstrated the increased quantity of cells expressing CD8+CD103+ in the lesional epidermis and CD4+103+ in the lesional dermis compared to the skin of healthy volunteers (Figure 5) $[9,13,19,20]$. Moreover, Kurihara et al. reported that the number of CD8+CD103+ TRM cells in psoriatic epidermis correlated with the thickness of the psoriatic plaque, i.e. the intensity of keratinocyte proliferation. They were capable of producing IFN- $\gamma$, IL-17A or IL-22 [4]. Additionally, the production of IL-17A was higher in patients with the longer duration of the disease [20]. The accumulation of epidermal CD8+ T cells may induce both keratinocyte hyperproliferation and papillomatosis (the parallel increase in CD8+ count and the intensity of Ki67 staining in keratinocytes) [13]. CD8+ cells infiltrating the epidermis are CD103+ and those are considered to be the proper TRM, while CD4+ in the dermis express CD103 to a low extent $[3,9]$. The present study also showed the expression of CD103 to be considerably lower (Figure 2).

There is a paucity of research assessing the dynamics of the changes of TRM count under the influence of implemented psoriasis treatment. The present study was performed to assess the location of typical TRM markers in the lesional epidermis and dermis at three time points for three different systemic therapies (MTX, anti-IL-17 and anti-TNF). It revealed that the intensity of the fluorescence of some antigens diminished throughout the therapies. The most rapid reduction in the expression of TRM-specific antigens was observed in case of anti-IL-17 therapy at week 4 of treatment, while with MTX and anti-TNF, the re- 

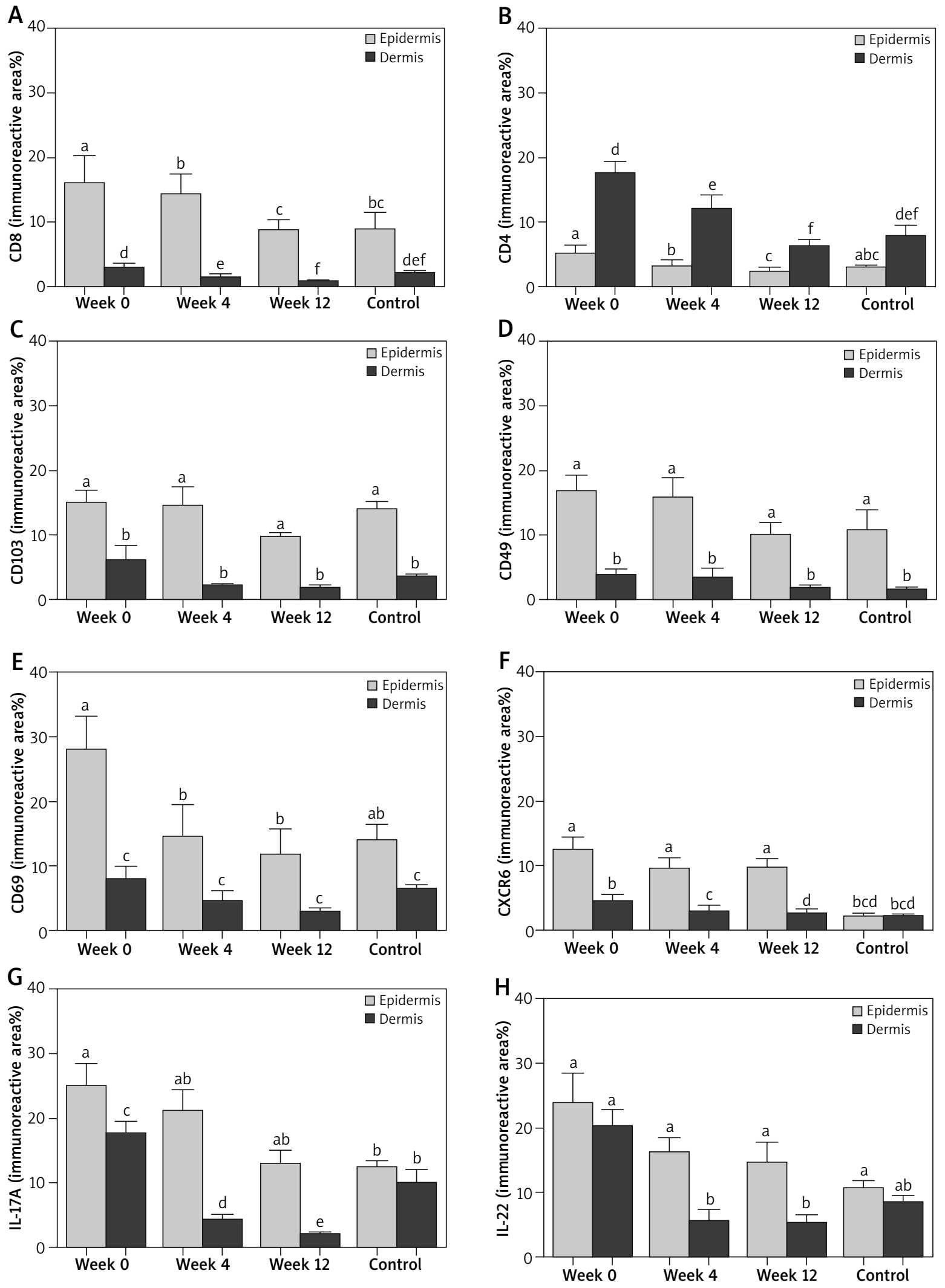

Figure 1. The immunoreactive area (\%) of CD8 ( $x \pm$ SEM) (A), CD4 ( $x \pm$ SEM) (B), CD103 ( $x \pm$ SEM) (C), CD49 ( $x \pm$ SEM) (D), CD69 $(x \pm$ SEM) $(E)$, CXCR6 $(x \pm$ SEM) $(F)$, IL-17A $(x \pm$ SEM) $(G)$ and IL-22 $(x \pm$ SEM) $(H)$ in the lesional skin of patients $(n=5$, dermis and epidermis) before (week 0 ) and during treatment (week 4 and week 12) with anti-interleukin-17 (anti-IL-17) in comparison with healthy controls ( $n=10$, dermis and epidermis). Bars with different letters are significantly different $(p<0.05)$ 

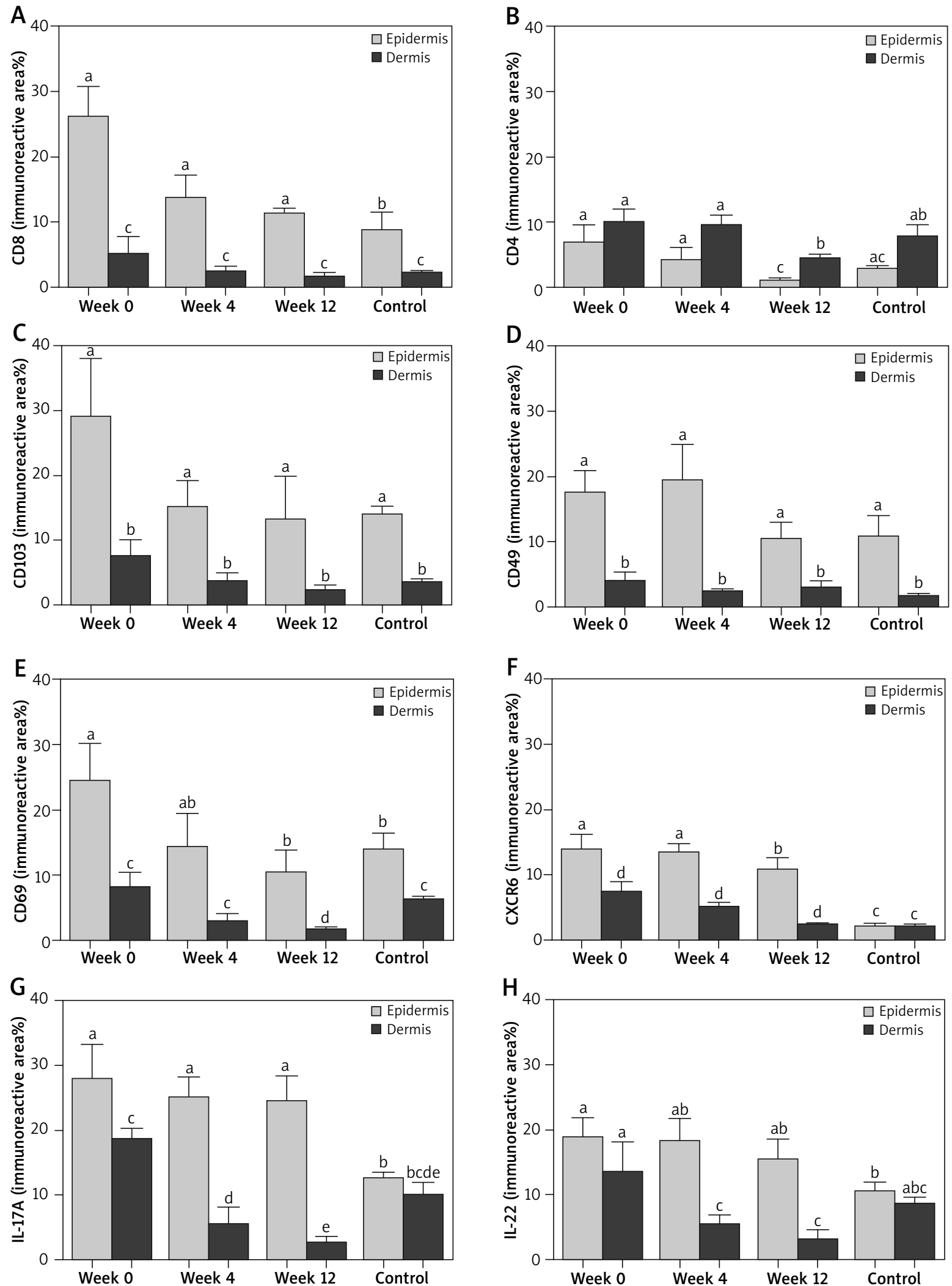

Figure 2. The immunoreactive area (\%) of CD8 $(x \pm$ SEM) (A), CD4 $(x \pm$ SEM) (B), CD103 $(x \pm$ SEM) (C), CD49 $(x \pm$ SEM) (D), CD69 $(x \pm$ SEM) (E), CXCR6 $(x \pm$ SEM) (F), IL-17A $(x \pm$ SEM) $(\mathrm{G})$ and IL-22 $(x \pm$ SEM) $(H)$ in the lesional skin of patients $(n=4)$, dermis and epidermis) before (week 0 ) and during treatment (week 4 and week 12) with anti-tumour necrosis factor $\alpha$ (TNF- $\alpha$ ) in comparison with healthy controls $(n=10$, dermis and epidermis). Bars with different letters are significantly different $(p<0.05)$ 

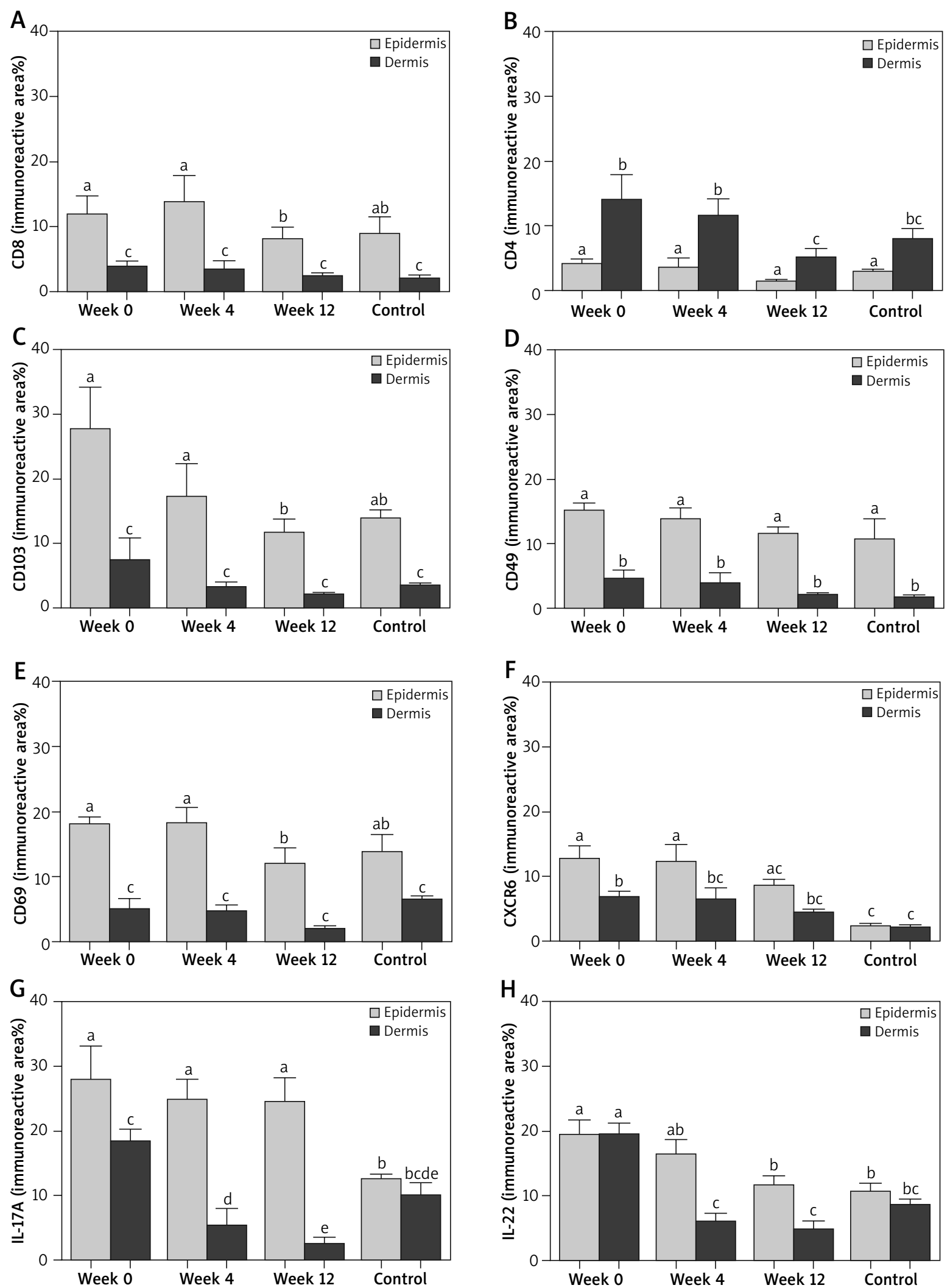

Figure 3. The immunoreactive area (\%) of CD8 $(x \pm$ SEM) (A), CD4 $(x \pm$ SEM) (B), CD103 ( $x \pm$ SEM) (C), CD49 ( $x \pm$ SEM) (D), CD69 $(x \pm$ SEM) (E), CXCR6 $(x \pm$ SEM) (F), IL-17A $(x \pm$ SEM) $(G)$ and IL-22 $(x \pm$ SEM) $(H)$ in the lesional skin of patients $(n=4$, dermis and epidermis) before (week 0$)$ and during treatment (week 4 and week 12) with methotrexate (MTX) in comparison with healthy controls $(n=10$, dermis and epidermis). Bars with different letters are significantly different $(p<0.05)$ 

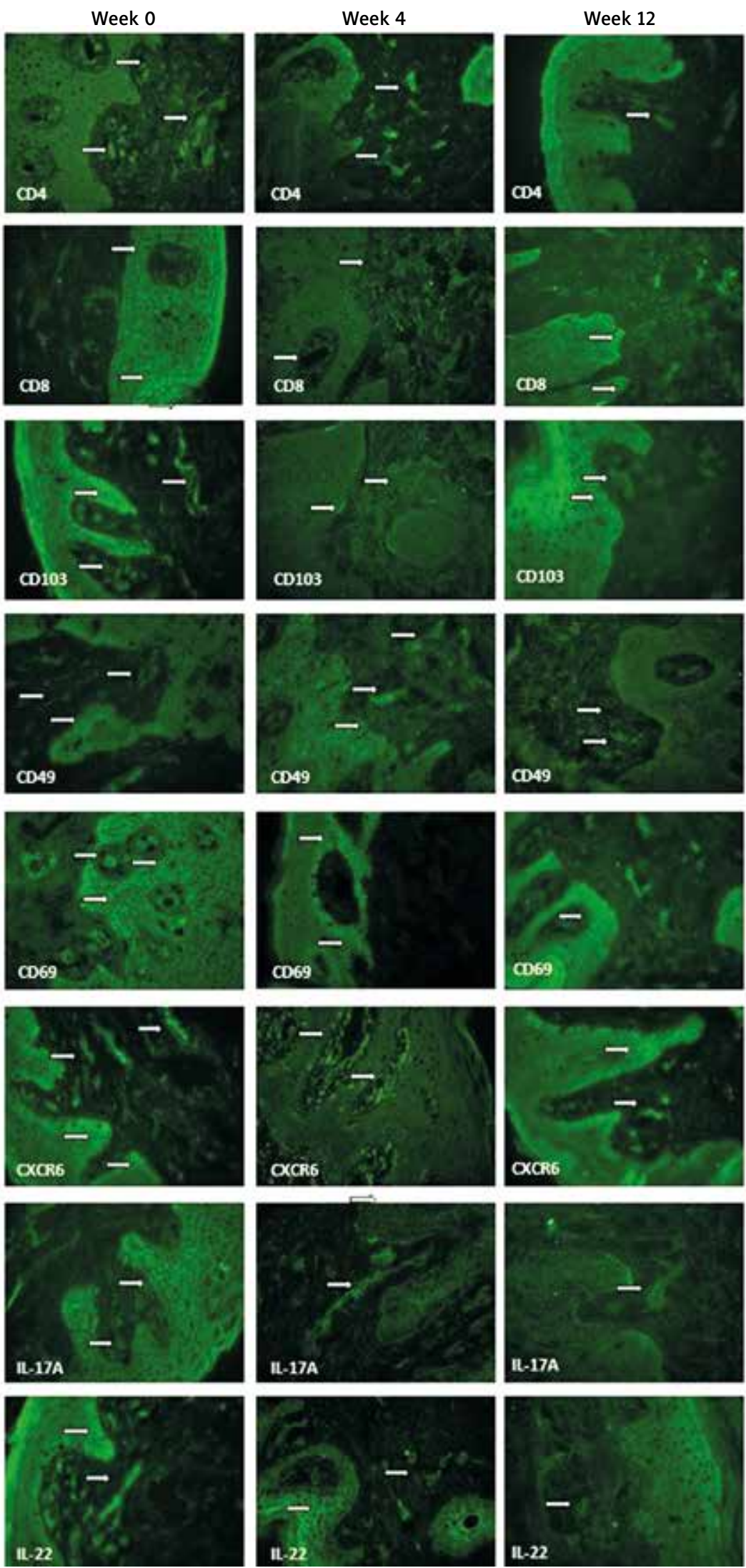

Figure 4. The skin location of CD8, CD4, CD103, CD49, CD69, CXCR6, IL-17A and IL-22 proteins (the representative sections) in the lesional skin (dermis and epidermis) for all the therapies. The proteins are marked in green (fluorescein). Magnification: $500 \times$ 
Agnieszka Owczarczyk-Saczonek, Marta Kasprowicz-Furmańczyk, Joanna Czerwińska, Magdalena Krajewska-Włodarczyk, Waldemar Placek

Table 1. Changes in the immunoreactive area (\%) of CD8, CD4, CD103, CD49, CD69, CXCR6, IL-17A and IL-22 proteins in patients during therapy with: anti-interleukin-17 (anti-IL-17), anti-tumour necrosis factor $\alpha$ (anti-TNF- $\alpha$ ), methotrexate (MTX) (week 0, 4 and 12). Bars with an asterisk show statistically significant differences $\left({ }^{*} p<0.05\right)$

\begin{tabular}{|c|c|c|c|c|c|c|}
\hline \multirow[t]{2}{*}{ Variable } & \multicolumn{2}{|c|}{ Week 0 (area \%) } & \multicolumn{2}{|c|}{ Week 4} & \multicolumn{2}{|c|}{ Week 12} \\
\hline & Epidermis & Dermis & Epidermis & Dermis & Epidermis & Dermis \\
\hline \multicolumn{7}{|l|}{ Anti-IL-17: } \\
\hline $\mathrm{CD} 8$ & 16.03 & 2.91 & $-12 \%$ & $-48 \%$ & $-44 \%^{*}$ & $-60 \% *$ \\
\hline CD4 & 5.06 & 17.37 & $-37 \%$ & $-31 \%$ & $-56 \%$ & $-64 \%^{*}$ \\
\hline CD103 & 15.02 & 5.96 & $-3 \%$ & $-61 \%$ & $-36 \%$ & $-69 \%$ \\
\hline CD49 & 16.94 & 3.71 & $-6 \%$ & $-9 \%$ & $-41 \%$ & $-49 \%$ \\
\hline CD69 & 28.00 & 7.72 & $-48 \%$ & $-44 \%$ & $-58 \% *$ & $-63 \%$ \\
\hline CXCR6 & 12.51 & 4.33 & $-24 \%$ & $-35 \%$ & $-22 \%$ & $-43 \% *$ \\
\hline IL-17A & 25.03 & 17.46 & $-15 \%$ & $-76 \%$ & $-47 \%$ & $-89 \%^{\star}$ \\
\hline IL-22 & 23.83 & 20.18 & $-32 \%$ & $-72 \%$ & $-38 \%$ & $-74 \%$ * \\
\hline \multicolumn{7}{|c|}{ Anti-TNF- $\alpha$ : } \\
\hline CD8 & 21.15 & 5.06 & $-46 \%$ & $-54 \%$ & $-54 \%$ & $-70 \%$ \\
\hline CD4 & 6.80 & 14.95 & $-39 \%$ & $-37 \%$ & $-85 \% *$ & $-70 \% *$ \\
\hline CD103 & 29.01 & 7.25 & $-48 \%$ & $-52 \%$ & $-54 \%$ & $-72 \%$ \\
\hline CD49 & 17.55 & 3.85 & $-10 \%$ & $-42 \%$ & $-41 \%$ & $-27 \%$ \\
\hline CD69 & 24.36 & 7.99 & $-41 \%$ & $-66 \%$ & $-57 \%^{*}$ & $-80 \% *$ \\
\hline CXCR6 & 13.77 & 7.18 & $-2 \%$ & $-30 \%$ & $-22 \%$ & $-67 \%$ \\
\hline IL-17A & 23.17 & 12.08 & $-5 \%$ & $-70 \%$ & $-19 \%$ & $-74 \% *$ \\
\hline IL-22 & 18.83 & 13.42 & $-2 \%$ & $-60 \%$ & $-18 \%$ & $-77 \%^{*}$ \\
\hline \multicolumn{7}{|l|}{ MTX: } \\
\hline CD8 & 9.73 & 3.86 & $16 \%$ & $-12 \%$ & $-28 \%$ & $-39 \%$ \\
\hline CD4 & 4.03 & 13.82 & $-14 \%$ & $-17 \%$ & $-69 \%$ & $-63 \% *$ \\
\hline CD103 & 27.64 & 7.25 & $-37 \%$ & $-57 \%$ & $-73 \%^{*}$ & $-58 \%$ \\
\hline CD49 & 14.96 & 4.38 & $-8 \%$ & $-13 \%$ & $-24 \%$ & $-56 \%$ \\
\hline CD69 & 18.05 & 4.94 & $1 \%$ & $-6 \%$ & $-66 \% *$ & $-40 \%$ \\
\hline CXCR6 & 12.68 & 6.62 & $-4 \%$ & $-4 \%$ & $-33 \%$ & $-34 \%$ \\
\hline IL-17A & 27.88 & 18.47 & $-10 \%$ & $-71 \%$ & $-12 \%$ & $-86 \% *$ \\
\hline IL-22 & 19.41 & 19.52 & $-15 \%$ & $-69 \%$ & $-61 \%^{*}$ & $-75 \%^{*}$ \\
\hline
\end{tabular}

sponse was observable no earlier than in week 12. It is unknown how long the treatment should last so as to eliminate TRM completely and prevent rapid relapses. Seemingly, it may vary depending on the therapy.

Cheuk et al. demonstrated the presence of $T$ cells producing both $\mathrm{IL}-22$ and $\mathrm{IL}-17$ in the epidermis and dermis of patients undergoing $n b-U V B$ therapy or patients treated with biological drugs [9]. The cells were significantly reduced in the biopsies collected after the effective treatment with nb-UVB (at least 25 irradiation sessions), after biological drug treatment (infliximab the median duration of treatment I was 4.5 years and with ustekinumab the median of treatment duration was 1.4 years). However, despite the normalization of the total CD8+ T lymphocyte count, the number of cells expressing
CD49a increased 5-fold in samples subjected to nb-UVB radiation and 16 -fold in samples collected from patients undergoing biological therapy [9].

Interestingly, ex vivo stimulation showed that the levels of CD4+ T lymphocytes producing IL-22 in the resolved lesions were similar or higher compared to active psoriasis and did not correlate with the number of years of treatment, indicating the maintenance of the effector function in the resolved lesions even after 6 years of treatment [9]. It may be explained by TRM resistance to destructive factors and apoptosis. The heterodimeric molecule of CD8+ $(\alpha E \beta 7)$ marker contains CD103 and $\beta 7$ subunits and plays a significant role in the stability of CD8+ TRM cells via increasing the expression of $\mathrm{BCl}-2$ which is characterized by its antiapoptotic properties [1, 4, 5]. Furthermore, active ef- 
Anti-IL-17

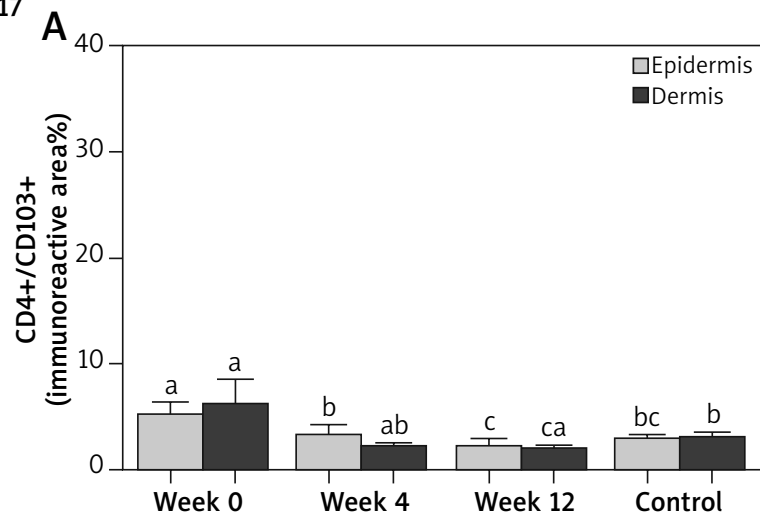

Anti-TNF- $\alpha$ C

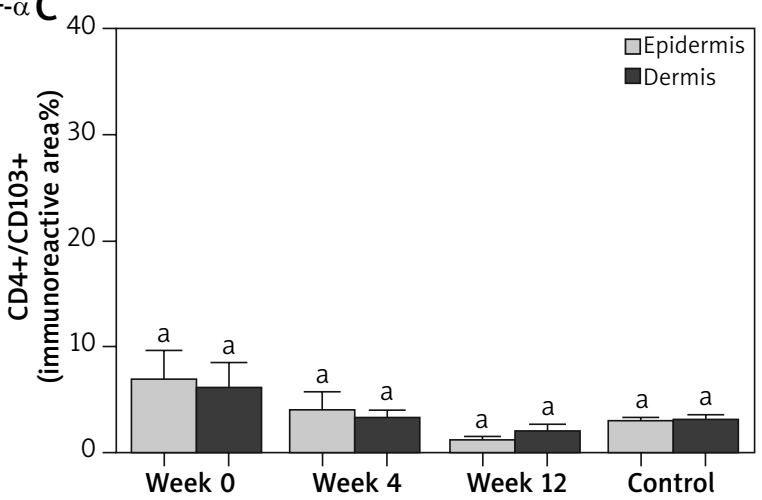

MTX

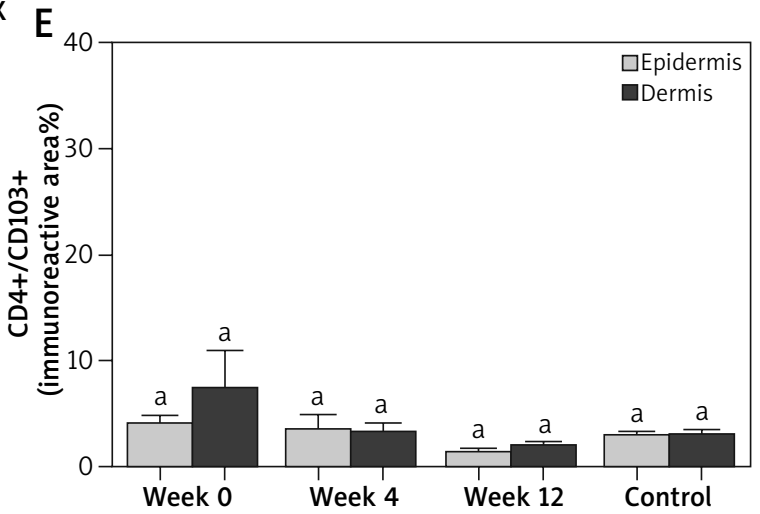

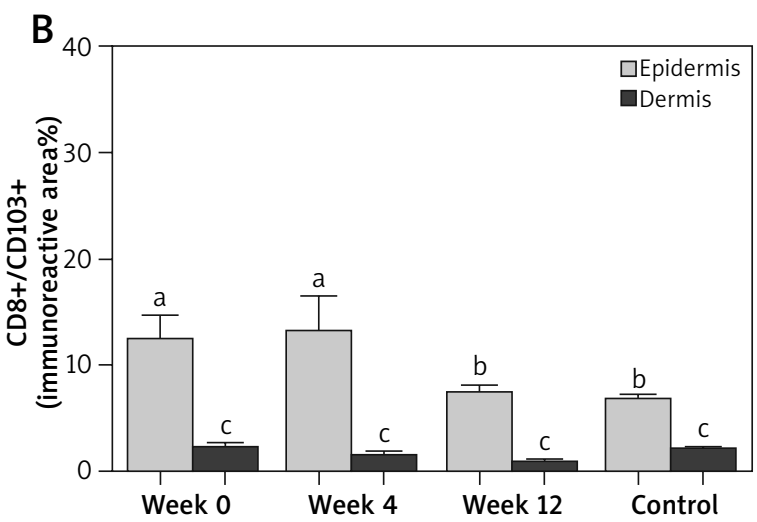
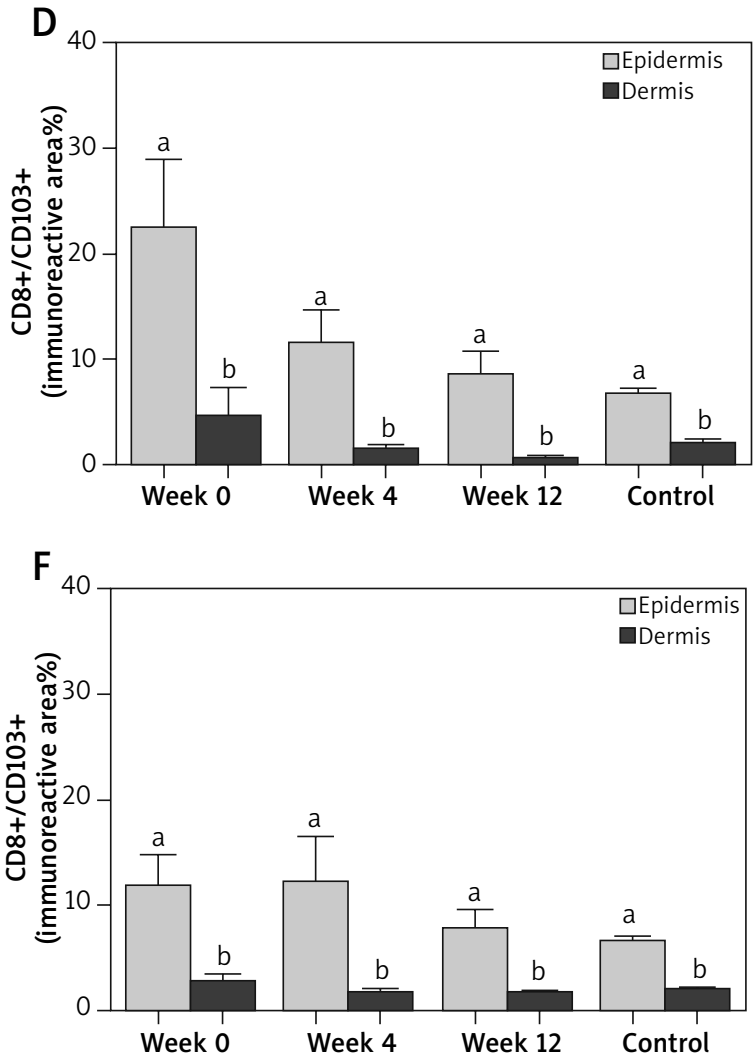

Figure 5. The immunoreactive area (\%) of CD8+CD103+ $(x \pm S E M)$ and CD4+CD103+ $(x \pm S E M)$ in the lesional skin of patients $(n=5$, dermis and epidermis) before (week 0$)$ and during treatment (week 4 and week 12) with anti-interleukin-17 (anti-IL-17), anti-tumour necrosis factor $\alpha$ (anti-TNF- $\alpha$ ), methotrexate (MTX) in comparison with healthy controls $(n=10$, dermis and epidermis). Bars with different letters are significantly different $(p<0.05)$

fector T cells participating in an inflammatory process may be converted into long-lasting TRM cells in the epidermis throughout disease resolution [1, 4, 5, 21].

Kurihara et al. investigated 10 patients who were treated systemically with cyclosporine, a phosphodiesterase-4 inhibitor (PDE4) or biological drugs for a year. The study showed that CD8+CD103+IL-17A+ TRM cells occurred more frequently in those patients. Interestingly, the investigators observed more CD8+ TRM than CD4+ TRM cells, which may be suggestive of their correlation with the duration of disease and not with the intensity of dermal lesions $[3,4]$. Our study also confirmed the resistance in the reduction of CD8+ TRM count in the epidermis during the treatment.

The latest study assessing the profile of lymphocytes in psoriatic eruptions after treatment with secukinumab (anti-IL-17) and guselkumab (anti-IL-23) demonstrated that both therapies reduced the number of inflammatory dendritic cells and CD4+CD49a-CD103- T cells. Interestingly, guselkumab reduced TRM count promoting regulatory 

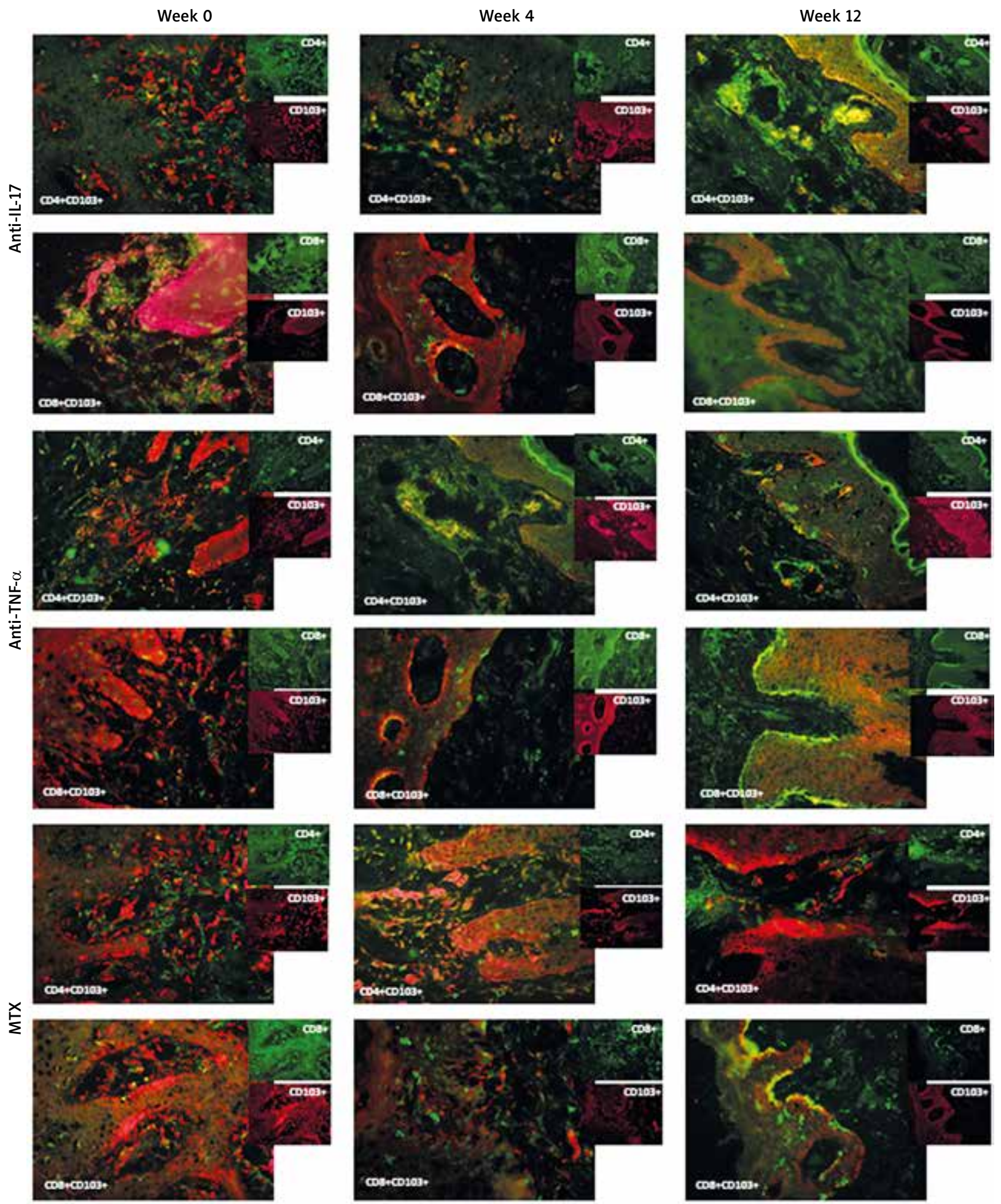

Figure 6. The skin location of CD8 (green - Alexa 488), CD4 (green - Alexa 488) and CD103 (red - Alexa 594) proteins (the representative sections) in the lesional skin of patients ( $n=5$ or $n=4$, dermis and epidermis) before (week 0$)$ and during treatment (week 4 and week 12) with: anti-interleukin-17 (anti-IL-17), anti-tumour necrosis factor $\alpha$ (anti-TNF- $\alpha$ ), methotrexate (MTX). Magnification: 500x 
T cells at the same time, while in case of secukinumab the situation was reversed. Furthermore, none of the drugs changed the quantity of IL-17A+IL-17F+/- CD4+ or CD8+ T cells [22].

It is a very important conclusion from the study, as longer remissions may be triggered after suppressing only $\mathrm{IL}-23$, and not IL-12, depending on the therapy. Interleukin-12 suppresses the production of $\gamma \delta \mathrm{T}(\gamma \delta \mathrm{T} 17)$ cells involved in IL-17 production. Moreover, according to the literature, IL-12 receptor signalling in keratinocytes initiates a protective, anti-inflammatory transcriptional program [23]. It explains more rapid relapses of the disease after blocking IL-17 compared to IL-23 inhibition $[17,22,24]$.

Gallais Sérézal et al. reported an increased expression of IL-17, which was still produced by CD8+ CD103+ TRM cells in the epidermis of successfully treated psoriasis patients. Trigger factors are responsible for the rapid activation of TRM in the epidermis, IL-17 production by TRM, which stimulates keratinocytes to release chemokines and recruit circulating leukocytes $[23,25,26]$. Our results were similar, i.e. the treatment resulted in a slight decrease in IL-17 expression in the epidermis.

It was noted in the present study that the reduction of the expression of TRM-specific antigens was observable predominantly in the lesional dermis, and not in the lesional epidermis. It may be due to several factors. There is a paucity of data concerning the penetration of systemic drugs into the epidermis - the absence of vasculature may contribute to poorer penetration of systemic drugs translating into poorer treatment outcomes. It is possible that the process of psoriatic lesion resolution in the epidermis and its gradual normalization are secondary to the reduction of inflammation in the skin, or epidermal TRM cells are more resistant to therapy. Therefore, a question arises: "How may topical drugs influence TRM quantity in psoriatic eruptions?" The above question will underlie our subsequent analysis.

We do realize that the small sample size constitutes a limitation of this study. Regrettably, the study was arduous and it required triple biopsy sampling at the same location of an eruption, which was difficult for the patient. The research is still ongoing and a longer observation time would be advisable (a repeat biopsy after 24 weeks).

Another weakness of the study is related to technical limitations. It would be indicated to use a higher number of markers in immunofluorescence in order to increase the specificity of the method.

\section{Conclusions}

The understanding of the mechanisms of psoriatic inflammation and the role of TRM may explain the key problems associated with the disease:

- the resistance of lesions to treatment and the reactivation of lesions at the same location,
- the isomorphic Koebner phenomenon,

- the appropriate duration of treatment, longer than the remission of eruptions, in order to suppress and reduce the quantity of TRM $[1,26,27]$.

The detailed elucidation of mechanisms responsible for the development/relapses of the disease may contribute to the implementation of more effective therapeutic protocols.

\section{Acknowledgments}

This research was funded by the University of Warmia and Mazury, grant number 320 61.610.006-110.

\section{Conflict of interest}

The authors declare no conflict of interest.

\section{References}

1. Owczarczyk-Saczonek A, Krajewska-Włodarczyk M, Kasprowicz-Furmańczyk M, et al. Immunological memory of psoriatic lesions. Int J Mol Sci 2020; 21: 625.

2. Watanabe R. Protective and pathogenic roles of resident memory T cells in human skin disorders. J Dermatol Sci 2019; 95: 2-7.

3. Tokura Y, Phadungsaksawasdi P, Kurihara K, et al. Pathophysiology of skin resident memory T cells. Front Immunol 2021; 11: 618897.

4. Kurihara K, Fujiyama T, Phadungsaksawasdi P, et al. Significance of IL-17A-producing CD8+CD103+ skin resident memory $T$ cells in psoriasis lesion and their possible relationship to clinical course. J Dermatol Sci 2019; 95: 21-7.

5. Mackay LK, Rahimpour A, Ma JZ, et al. The developmental pathway for CD103(+)CD8+ tissue-resident memory T cells of skin. Nat Immunol 2013; 14: 1294-301.

6. Walsh DA, Borges da Silva H, Beura LK, et al. The functional requirement for CD69 in establishment of resident memory CD8 ${ }^{+} T$ cells varies with tissue location. J Immunol 2019; 203: 946-5.

7. Zhang Y, Lin Y, Wang L, et al. CD $8 \alpha \alpha+T$ Cells exert a proinfammatory role in patients with psoriasis. Skin Health Dis 2021; 1: e64.

8. Samat AAK, van der Geest J, Vastert SJ, et al. Tissue-resident memory $T$ cells in chronic inflammation-local cells with systemic effects? Cells 2021; 10: 409.

9. Cheuk S, Schlums H, Gallais Sérézal I, et al. CD49a expression defines tissue-resident $C D 8^{+} \mathrm{T}$ cells poised for cytotoxic function in human skin. Immunity 2017; 46: 287-300.

10. Dijkgraaf FE, Kok L, Schumacher TNM. Formation of tissueresident $\mathrm{CD}^{+}$T-cell memory. Cold Spring Harb Perspect Biol 2021; 13: a038117.

11. Kumar B, Ma W, Miron M, et al. Human tissue-resident memory $T$ cells are defined by core transcriptional and functional signatures in lymphoid and mucosal sites. Cell Rep 2017; 20: 2921-34.

12. Zaid A, Mackay LK, Rahimpour A, et al. Persistence of skinresident memory T cells within an epidermal niche. Proc Natl Acad Sci USA 2014; 111: 5307-12.

13. Di Meglio P, Villanova F, Navarini AA, et al. Targeting CD8(+) T cells prevents psoriasis development. J Allergy Clin Immunol 2016; 138: 274-6. 
14. Gallais Sérézal I, Classon C, Cheuk S, et al. Resident T cells in resolved psoriasis steer tissue responses that stratify clinical outcome. J Invest Dermatol 2018; 138: 1754-63.

15. Ritchlin C. Tissue-resident memory T cells: sequestered immune sensors and effectors of inflammation in spondyloarthritis. Arthr Rheumatol 2020; 72: 379-82.

16. Huang YW, Tsai TF. Remission duration and long-term outcomes in patients with moderate-to-severe psoriasis treated by biologics or tofacitinib in controlled clinical trials: a 15-year single-center experience. Dermatol Ther (Heidelb) 2019; 9: 553-69.

17. Masson Regnault M, Konstantinou MP, Khemis A, et al. Early relapse of psoriasis after stopping brodalumab: a retrospective cohort study in 77 patients. J Eur Acad Dermatol Venereol 2017; 31: 1491-6.

18. Wang XY, Zhang CL, Wang WH. Time to relapse after treatment withdrawal for different biologics used to treat plaque psoriasis. Chin Med J 2020; 133: 2998-3000.

19. Diani M, Altomare G, Reali E. T helper cell subsets in clinical manifestations of psoriasis. J Immunol Res 2016; 2016: 7692024.

20. Vo S, Watanabe R, Koguchi-Yoshioka H, et al. CD8 resident memory $T$ cells with interleukin 17A producing potential are accumulated in disease-naïve nonlesional sites of psoriasis possibly in correlation with disease duration. Br J Dermatol 2019; 181: 410-2.

21. Casey KA, Fraser KA, Schenkelm JM, et al. Antigen-independent differentiation and maintenance of effector-like resident memory T cells in tissues. I Immunol 2012; 188: 4866-75.

22. Mehta H, Mashiko S, Angsana J. Differential changes in inflammatory mononuclear phagocyte and T-cell profiles within psoriatic skin during treatment with guselkumab vs. secukinumab. J Invest Dermatol 2021; 141: 1707-18e9.

23. Benezeder T, Wolf P. Resolution of plaque-type psoriasis: what is left behind (and reinitiates the disease). Semin Immunopathol 2019; 41: 633-44.

24. Reich K, Armstrong AW, Langley RG, et al. Guselkumab versus secukinumab for the treatment of moderate-to-severe psoriasis (ECLIPSE): results from a phase 3, randomised controlled trial. Lancet 2019; 394: 831-9.

25. Gallais Sérézal I, Hoffer E, Ignatov B, et al. A skewed poo of resident $T$ cells triggers psoriasis-associated tissue responses in never-lesional skin from patients with psoriasis. J Allergy Clin Immunol 2019; 143: 1444-54.

26. Gallais Sérézal I, Cheuk S, Martini E, et al. Cellular scars and local crosstalk in relapsing psoriasis: an example of a skin sticking disease. Scand J Immunol 2020; 92: e12953.

27. Chen L, Shen Z. Tissue-resident memory T cells and their biological characteristics in the recurrence of inflammatory skin disorders. Cell Mol Immunol 2019; 17: 64-75. 